\title{
Financial Influences and Scale-Free Networks
}

\author{
Nitin Arora ${ }^{1}$, Babu Narayanan ${ }^{2}$, and Samit Paul ${ }^{2}$ \\ 1 Google Inc., Bangalore 560001, India \\ nitinarora@google. $\mathrm{com}^{\star}$ \\ 2 GE Global Research, \\ John F. Welch Technology Centre, \\ Bangalore 560066, India \\ babu.narayanan@ge.com, samit.paul@geind.ge.com
}

\begin{abstract}
We consider the problem of analyzing influences in financial networks by studying correlations in stock price movements of companies in the $S \& P 500$ index and measures of influence that can be attributed to each company. We demonstrate that under a novel and natural measure of influence involving cross-correlations of stock market returns and market capitalization, the resulting network of financial influences is Scale Free. This is further corroborated by the existence of an intuitive set of highly influential hub nodes in the network. Finally, it is also shown that companies that have been deleted from the $S \& P 500$ index had low values of influence.
\end{abstract}

\section{Introduction}

In a stock market, individual companies are influenced by each other. The nature and measure of the influence of a company on another depends on various factors including their sectors of operation, direct buyer-seller relation, acquisition, etc. A quantity that measures the direct influence among companies is the relationship between their stock price changes. This can be measured using the pairwise correlations of the two stock prices averaged over a time window. Analysis of such correlations, usually using matrices, is a well studied topic in computational finance. In this paper, following Kim et al [1, we pursue a graph theoretic approach to obtain interesting structural properties in this correlation matrix. A weighted discrete graph is constructed with the nodes representing the companies and the weight of the edge between two companies equals the correlation. The analysis is carried out on this graph.

Scale free graphs and networks have generated a lot of interest lately. A graph is said to be scale free if the degree sequence of the graph follows a power law - the probability that a vertex has $\mathrm{k}$ neighbors is proportional to $k^{\gamma}$. Scale free networks show up in a large number of real world scenarios, especially large complex networks. For example, it is shown in [2] that the scale free property applies to the world wide web graph consisting of documents as vertices, two

\footnotetext{
* The work of the first author was carried out while he was visiting the John F. Welch

Technology Centre, Bangalore 560066, India.
} 
documents being connected if a hyperlink points one document to the other. Many social and business networks are also scale free 3 . In nature, one finds that cellular metabolic networks and protein interaction networks are scale free 4, 5. Scale free networks have some interesting properties. There are a few nodes with very high degree of connectivity - called hubs. Most nodes on the other hand have very few neighbors. A computer network that is scale free would be resistant to random security attacks, but would be vulnerable to planned attacks on the hubs, see [6]. A popular theoretical model for the evolution of scale free networks uses the notion of preferential attachment and growth - a new vertex that is added attaches itself, with higher probability, to vertices that are already highly connected. See [7,8, 9].

In this paper, we study the network of companies in the $S \& P 500$ index. [1] used cross correlations as the metric and defined the influence of a company as the absolute sum of the crosscorrelations involving the company and conjectured that the resulting influence graph would be scale free. Here, note that the graph is a weighted fully connected graph and scale free refers to the existence of a power law governing the set of influences. Our analysis of similar data shows that under the measure in [1] the companies with the largest influence tended to all come out of the same sector, namely, semiconductor sector and included many relatively smaller companies. We present a novel alternate measure involving cross correlations and market capitalization under which the S\&P500 influence network is scale free. Also, the list of companies with large influence is intuitive. We also examine the companies that have been deleted from the index in 2004-2005 and observe that the deleted ones indeed had low influence over a period of time.

The rest of the paper is organized as follows. Section 2 provides the details of the data used. Section 3 discusses the different measures of influence and analyzes the results obtained. Finally, section 4 summarizes the results and provides direction for further work.

\section{Data}

The daily stock price data, adjusted for splits and dividends, for $S \& P 500$ companies from 1994 to 2005 was downloaded from the website www.finance.yahoo.com. Only companies that remained in the index from 1994 to 2004 were considered for the main part of the analysis. The final experiments were done for 416 companies that remained after data cleaning. The market capitalization values for these companies were downloaded for a particular day in July 2005 as a representative value. We calculated the cross correlations and influence values over the period 1994 to 2005 for various window sizes ranging from 3 months to 4 years. In this paper, we present some representative results.

\section{Analysis and Results}

Let the stock price of company $i(i=1, \ldots, N)$ at time $t$ be $Y_{i}(t)$. The return of the stock price after a time-interval $\Delta t$ is given by 


$$
S_{i}(t)=\ln Y_{i}(t+\Delta t)-\ln Y_{i}(t),
$$

meaning the geometrical change of $Y_{i}(t)$ during the interval $\Delta t$. We take $\Delta t=1$ day for the following analysis. The cross-correlations between individual stocks are considered in terms of a matrix $\mathrm{C}$, whose elements are given as

$$
C_{i, j}=\frac{<S_{i} S_{j}>-<S_{i}><S_{j}>}{\sqrt{\left(<S_{i}^{2}>-<S_{i}>^{2}\right)\left(<S_{j}^{2}>-<S_{j}>^{2}\right)}},
$$

where the brackets mean a temporal average over the period we studied. Then $C_{i, j}$ can vary between $[-1,1]$, where $C_{i, j}=1(-1)$ means that two companies $i$ and $j$ are completely positively(negatively) correlated, while $C_{i, j}=0$ means that they are uncorrelated. It is known that the distribution of the coefficients $\left\{C_{i, j}\right\}$ is a bellshaped curve, and the mean value of the distribution is slowly time-dependent, while the standard deviation is almost constant. The timedependence of the mean value might be caused by external economic environments such as bank interest, inflation index, exchange rate, etc, which fluctuates from time to time. To extract intrinsic properties of the correlations in stock price changes, we look at the following quantity as in [1,

$$
G_{i}(t)=S_{i}(t)-\frac{1}{N} \sum_{i} S_{i}(t),
$$

where $G_{i}(t)$ indicates the relative return of a company $i$ to its mean value over the entire set of $N$ companies at time $t$. The cross-correlation coefficients are redefined in terms of $G_{i}$,

$$
w_{i, j}=\frac{<G_{i} G_{j}>-<G_{i}><G_{j}>}{\sqrt{\left(<G_{i}^{2}>-<G_{i}>^{2}\right)\left(<G_{j}^{2}>-<G_{j}>^{2}\right)}} .
$$

To check that the distribution $P(w)$ is time-independent, 1] looked at $P(w)$ by taking temporal average in equation (4) over each year from 1993 to 1997 . The distribution of $\left\{w_{i, j}\right\}$ for the period 1994-1997 are shown in Figure 1 and match the distribution obtained by [1].

Let us now look at the weighted graph defined as follows - the vertices of the graph are individual companies, each pair $(i, j)$ of vertices has an edge between them with an associated weight $e_{i, j}$. The value $e_{i, j}$ represents the influence that the companies exert on each other. The total influence strength $s_{i}$ of company $i$ can then be defined as the sum of the weights on the edges incident upon the vertex $i$, that is,

$$
s_{i}=\sum_{j} e_{i, j},
$$

What will be a good way to calculate $e_{i, j}$ ? Does it depend only on the crosscorrelation coefficients? Should the influence of company $i$ on $j$ be the same as that of $j$ on $i$ ? [1] took $e_{i, j}=w_{i, j}$ in the above definition of influence strength (note that it is symmetric). For them, 


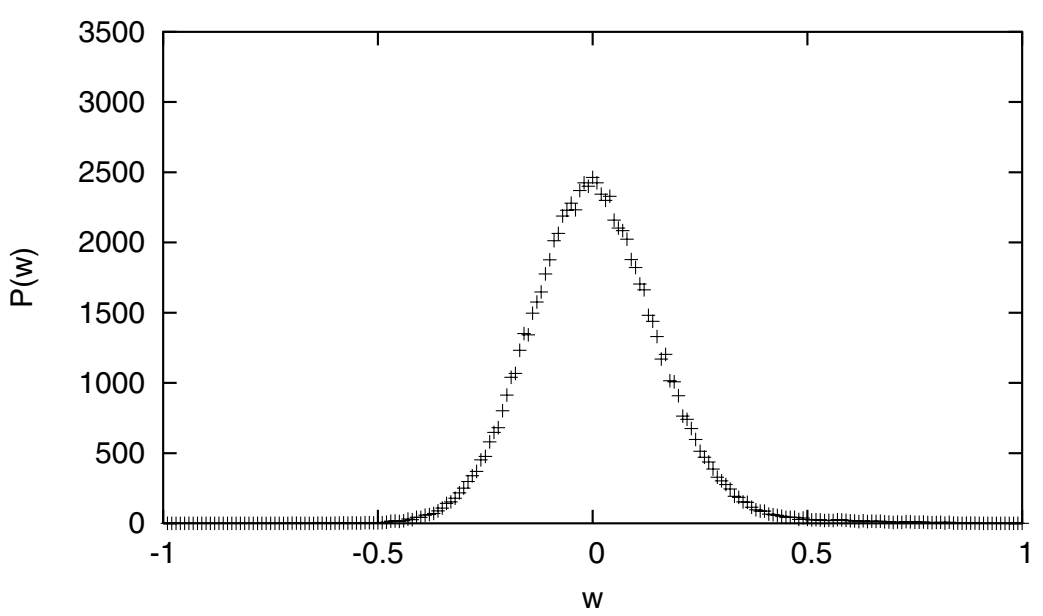

Fig. 1. Plot of the distribution of the cross correlation coefficients over the period 1994 to 1997. $P(w)$ denotes the number of pairs of companies with cross correlation coefficient $w \pm 0.005$.

$$
s_{i}=\sum_{j \neq i} w_{i, j} .
$$

We obtained the values $w_{i, j}$ using stock prices of $S \& P 500$ companies over a period of 4 years(1994-1997). It was observed that the definition in (6) assigns the highest values of influence strengths to companies such as AMAT, PMCS, KLAC, XLNX, LSI - surprisingly all of them in the semiconductor sector, while the companies such as GE, JNJ, PG, XOM and MSFT all landed in lower influence region (the symbols used for companies can be looked up for company name at www.finance.yahoo.com). Upon analysis, it was inferred that this was caused by a strong correlation between semiconductor companies, which in some cases was as high as 0.6 , a very rare value otherwise. The typical graph of the distribution $P(|s|)$ of the influence strength $|s|$ looked like in Figure 2 .

Above observations suggest using the values of market capitalizations of individual companies while calculating the influence strengths. We first attempted the following formula to calculate $s_{i}$,

$$
s_{i}=\sum_{j} w_{i, j} \times M_{j}
$$

where $M_{j}$ is the market capitalization of company $j$. That is, the influence of two companies $A$ and $B$ on the market should not be the same value if $A$ exerts high influence on bigger companies but $B$ exerts high influence on smaller companies. Also, for the case $j=i\left(w_{i, j}=1\right)$, we are adding the market capitalization of company $i$ to $s_{i}$ which makes intuitive sense. This definition of influence strength moved many of the bigger companies to the top. The most influential companies given by this formula are shown in Table1. The influence strength was calculated using a window of size two years(2003-04). 


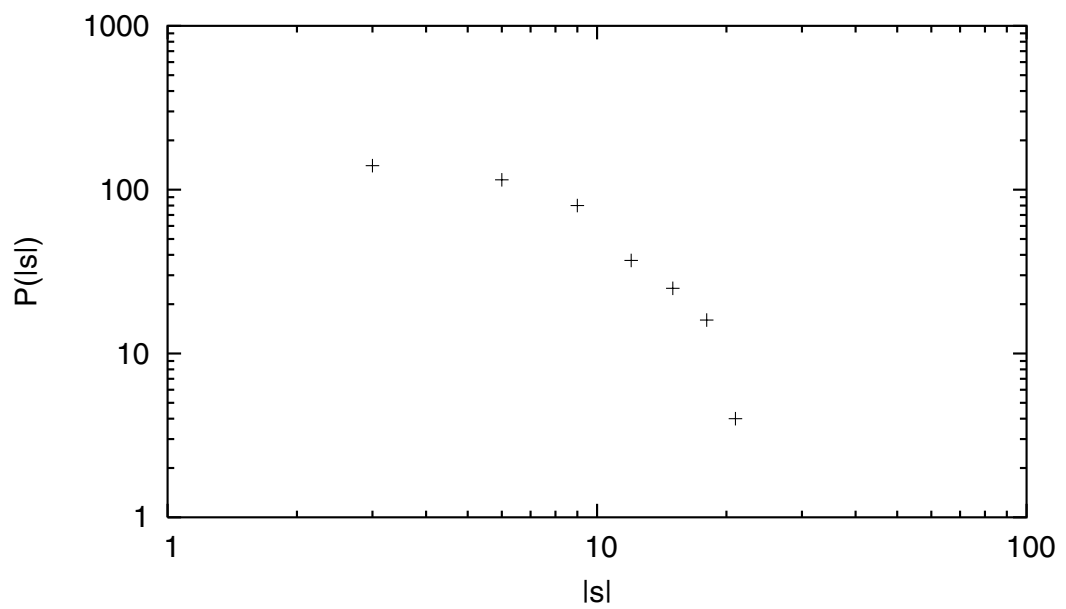

Fig. 2. Plot of the influence strength distribution $P(|s|)$ versus the absolute value of the influence strength $|s|$ as defined in [1. (in double-logarithmic scales) $P(|s|$ ) denotes the number of companies with influence $s \pm 1.5$.

Table 1. Top influential companies under equation (7)

\begin{tabular}{|c|c|c|}
\hline Symbol & Mkt Cap $(\mathrm{B} \$)$ & $\operatorname{Infl} \operatorname{Str}(\mathrm{B} \$)$ \\
\hline WFC & 102.66 & 1069.33 \\
\hline PG & 130.96 & 1047.74 \\
\hline XOM & 376.28 & 1032.79 \\
\hline JNJ & 192.66 & 980.272 \\
\hline BUD & 35.67 & 964.955 \\
\hline BAC & 181.41 & 938.318 \\
\hline PMCS & 1.73 & 913.625 \\
\hline GE & 368.24 & 875.932 \\
\hline C & 241.69 & 842.594 \\
\hline PEP & 88.05 & 832.869 \\
\hline
\end{tabular}

Table 2. Top influential companies under equation (8)

\begin{tabular}{|c|c|c|}
\hline Symbol & Mkt Cap(B $\$)$ & $\operatorname{Infl} \operatorname{Str}(\mathrm{B} \$)$ \\
\hline XOM & 376.28 & 720.45 \\
\hline PG & 130.96 & 590.82 \\
\hline JNJ & 192.66 & 589.969 \\
\hline BAC & 181.41 & 555.194 \\
\hline GE & 368.24 & 552.366 \\
\hline WFC & 102.66 & 539.617 \\
\hline C & 241.69 & 509.567 \\
\hline WMT & 206.51 & 471.956 \\
\hline PEP & 88.05 & 404.739 \\
\hline PFE & 199.04 & 392.209 \\
\hline
\end{tabular}

\subsection{Scale Free Network}

We now propose our final measure. To motivate, let us calculate the influence of company $i$ on the market in two steps. The symmetric measure of cross correlation $w_{i, j}$ between $i$ and $j$ can not be interpreted as influence of $i$ on $j$ as well as the influence of $j$ on $i$. This is too simplistic. Suppose a company $i$ with market capitalization $M_{i}(\sim 1 B)$ is supplying a raw material to a manufacturer $j$ with market capitalization $M_{j}(\sim 300 B)$. Suppose further that the stock price of $i$ is correlated to that of $j$ with $w_{i, j}(\sim 0.2)$. We propose that the influence exerted by $i$ on $j$, denoted $\operatorname{Inf} l(i \rightarrow j)$, is $w_{i, j} \times \frac{M_{i}}{M_{i}+M_{j}}$. Similarly define the influence by $j$ on $i$. Now to calculate the influence of $i$ on the market we will sum 


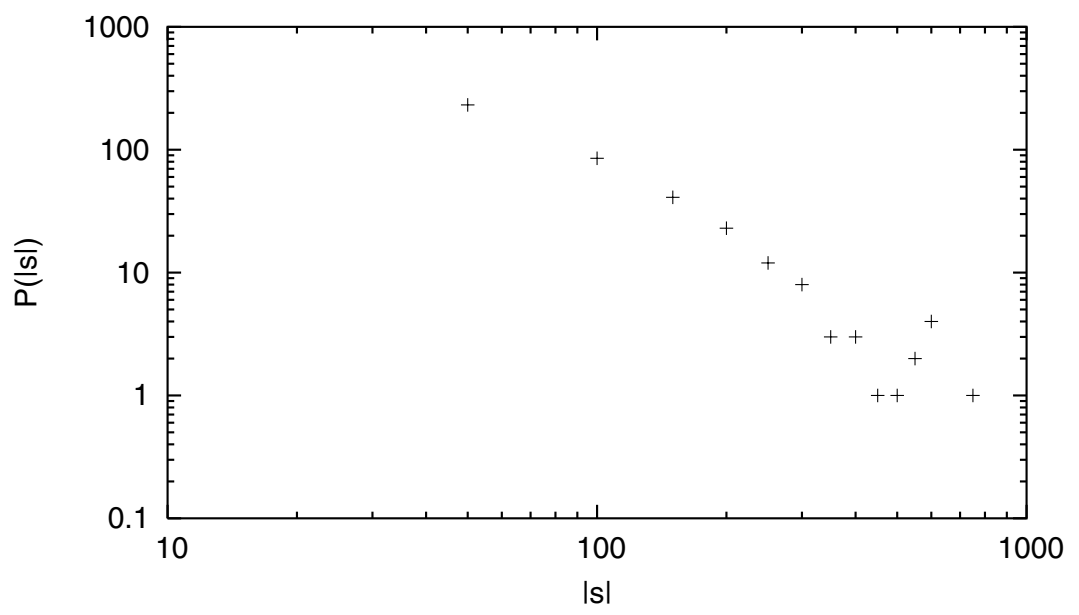

Fig. 3. Plot of the influence strength distribution $P(|s|)$ versus the absolute value of the influence strength $|s|$ as given by equation (8) (in double-logarithmic scales). We looked at the stock returns for two years 2003-2004. $P(|s|)$ denotes the number of companies with influence $s \pm 25$

over all companies $j$ the value of influence of $i$ on $j$ multiplied by the market capitalization of $j$. This gives

$$
s_{i}=\sum_{j} \operatorname{Infl}(i \rightarrow j) \times M_{j}=\sum_{j} w_{i, j} \times \frac{M_{i} M_{j}}{M_{i}+M_{j}} .
$$

This definition of influence strength gives a much more satisfactory ranking to companies. The top 10 companies are shown in Table 2.

Moreover, the plot of influence strength distribution $P(|s|)$ as a function of $|s|$ follows a power-law, $P(|s|) \sim|s|^{-\delta}$, with $\delta \approx 2.2$. See Figure 3. Thus the network of influences among these companies is indeed scale free. The outliers towards the high influence region may be attributed to extremely dominant companies in separate sectors. In particular, note that $P(|s|)$ in these cases is small.

Comparison of the Walt Disney Co and Texas Instruments Inc(both with market capitalization $\sim 50 B$ ) calculated over various 6 month windows between 2001 and 2004 shows that our measure does not give undue importance to market capitalization (See Figure 4).

\subsection{Deletion}

Finally, we looked at some companies that were deleted from the $S \& P 500$ recently, namely, American Greetings(Apr '04), Thomas and Belts(Aug '04), Worthington Industries(Dec '04) and Deluxe Corp.(Dec '04). Figure 5] shows the influence strength of these companies for the eight half-yearly periods between Feb 2001 and Feb 2005. A downward trend in their influence strengths just be- 


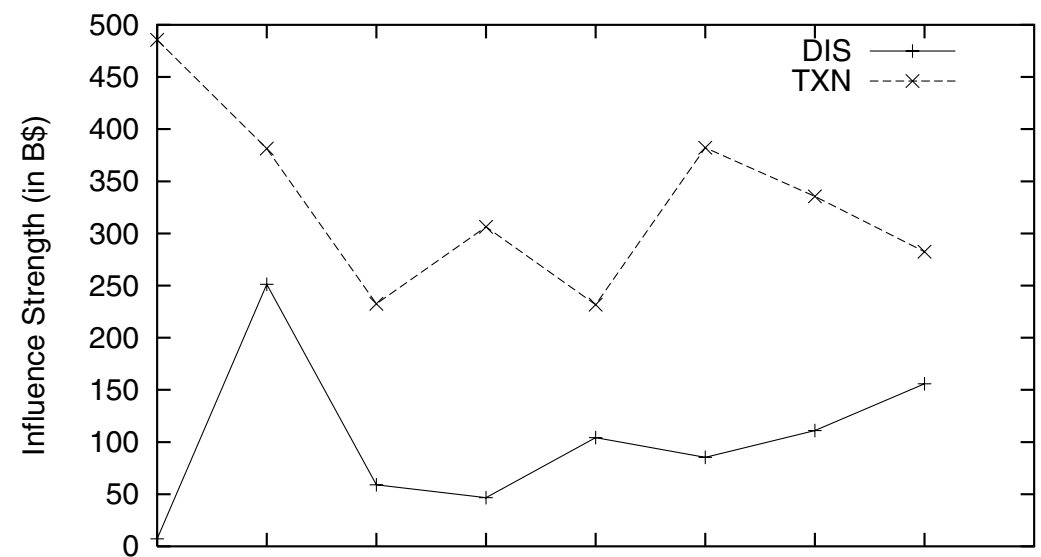

Feb 01 Aug 01 Feb 02 Aug 02 Feb 03 Aug 03 Feb 04 Aug 04

Fig. 4. Comparison of the influence strengths of DIS and TXN between 2001 and 2004

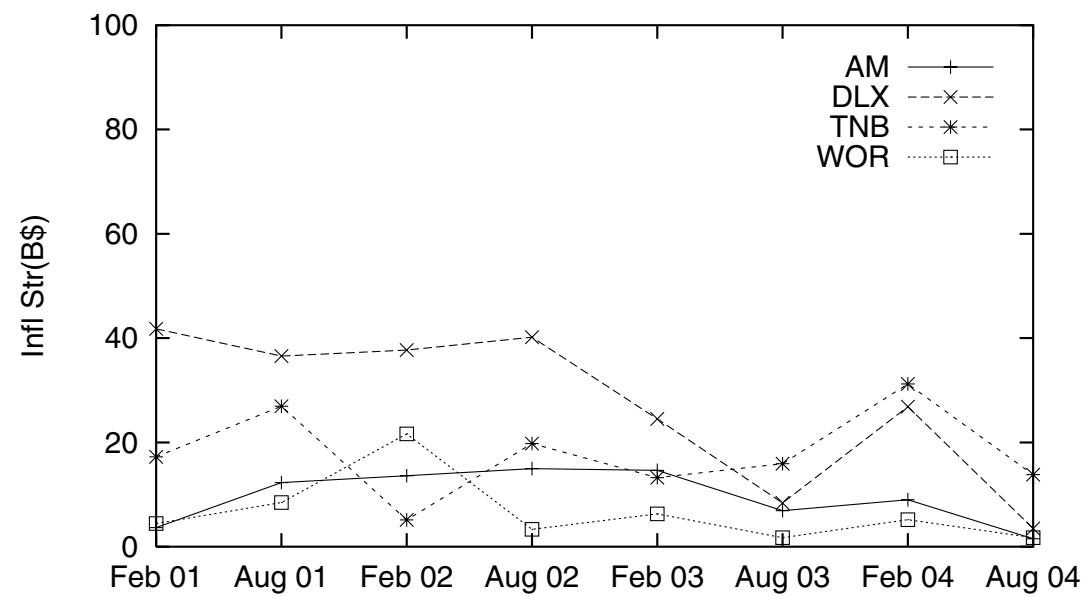

Fig. 5. Influence strengths of AM, DLX, TNB and WOR between Feb 2001 and Feb 2005. These were removed from the list of S\&P500 companies in 2004. Note the low values relative to the companies in Table 2 having influence $>350$.

fore being removed from the $S \& P 500$ index is seen. Also these influence strength values are substantially less than most of the other companies.

\section{Conclusion}

We demonstrate a weighted scale free network of influences that exists among $S \& P 500$ companies. We have come up with a measure for the influence of a company on another as well as on the whole market by utilizing cross-correlations 
on movements of stock prices as well as market capitalization. Our definition for the influence strength is observed to intuitively give a satisfactory ranking to the $S \& P 500$ companies and can also explain the deletion of companies from this index in the period from 2004 to 2005. Some open questions include analysis of other financial networks, analysis of the theoretical models for scale free networks in this context. An important problem is to derive methods of risk analysis for finance, supply chain and other domains incorporating concepts from the theory of scale free networks.

\section{References}

1. Kim, H.J., Lee, Y., Kim, I.M., Kahng, B.: Weighted scale-free network in financial correlations. Physical Society of Japan 71 (2002) 2133-2136

2. Albert, R., Jeong, H., Barabási, A.L.: Scale-free characteristics of random networks: The topology of the world-wide web (2000)

3. Barabási, A.L., Bonabeau: Scale-free networks. SCIAM: Scientific American 288 (2003)

4. $\mathrm{Hu}, \mathrm{X}$.: Mining and analysing scale-free protein-protein interaction network. Int. J. of Bioinformatics Research and Applications 1 (2005) 81-101

5. Jeong, H., Tomber, B., Albert, R., Oltvai, Z.N., Barabási, A.L.: The large-scale organization of metabolic networks. Nature 407 (2000) 651-654

6. Albert, R., Jeong, H., Barabási, A.L.: Error and attack tolerance of complex networks. NATURE: Nature 406 (2000)

7. Bollobás, B., Riordan: Mathematical results on scale-free random graphs. In Bornholdt, Schuster, eds.: Handbook of Graphs and Networks: From the Genome to the Internet. (2003)

8. Bollobás, B., Riordan, Spencer, Tusnady: The degree sequence of a scale-free random graph process. RSA: Random Structures \& Algorithms 18 (2001)

9. Bollobás, B., Borgs, C., Chayes, J.T., Riordan, O.: Directed scale-free graphs. In: SODA. (2003) 132-139 